\title{
Stress na gestação e no puerpério: uma correlação com a depressão pós-parto
}

\author{
Stressinpregnancyandpuerperium:acorrelationwithpostpartum \\ depression
}

Palavras-chave

Gravidez

Período pós-parto

Estresse psicológico

Depressão pós-parto

Saúde mental

Keywords

Pregnancy

Postpartum period

Stress, psycological

Postpartum, depression

Mental health
Correspondência:

Olga Maria Piazentin Rolim Rodrigues Av. Eng. Edmundo Carriio Coube, 14-01, B Vargem Limpo - CEP: 13033-360

Bauru (SP), Brasil

Recebido

04/08/2011

\section{Resumo}

OBJETIVO: Descrever e comparar as fases do stress de primigestas no terceiro trimestre de gestação e no pós-parto e correlacioná-las à ocorrência de depressão pós-parto (DPP). MÉTODOS: A pesquisa foi constituída de duas etapas, caracterizando-se como pesquisa longitudinal. Na Ełapa 1, participaram 98 primigestas e na Etapa 2, 64 delas. $\mathrm{Na}$ Etapa 1, a coleta de dados aconteceu no terceiro trimestre de gestação e, na Etapa 2, no mínimo 45 dias após o parto. Na Etapa 1 aplicou-se o Inventário de Sintomas de Stress de Lipp (ISSL) e uma Entrevista Inicial para caracterização da amostra. Na Ełapa 2, aplicou-se novamente o ISSL e também a EPDS (Escala de Edimburgo). Os dados foram analisados usando o programa estatístico SPSS for Windows ${ }^{\circledR}$, versão 17.0. As análises estatísticas efetuadas foram - Teste $t$ de Student e p de Spearman. RESULTADOS: No terceiro trimestre, 78\% das participantes apresentaram sinais significativos para stress e, no puerpério, $63 \%$ manifestaram, apresentando diferença significativa entre o stress manifestado no terceiro trimestre e no puerpério $(t=2,20 ; p=0,03)$. Observou-se, também, correlação entre o stress apresentado tanto na gestação como no puerpério e a manifestação de DPP ( $p<0,001)$. CONCLUSÃO: Tanto na gestação como no puerpério mais da metade das mulheres apresentam sinais significativos para stress. Entretanto, a frequência da manifestação dos sintomas significativos de stress na gestação foi superior à frequência apresentada no puerpério. Tais resultados parecem guardar uma estreita relação com a manifestação de DPP, indicando relação entre stress e DPP.

\section{Abstract}

PURPOSE: To describe and compare the phases of stress of primiparae in the third trimester of pregnancy and postpartum, associating them with the occurrence of postpartum depression. METHODS: The study consisted of two stages (Stage 1 and Stage 2), characterized as longitudinal research. Ninety-eight primiparae participated in Stage 1, and 64 of them participated in Stage 2. In Stage 1, data were collected in the third trimester of pregnancy, and in Stage 2, at least 45 days after delivery. The Stress Symptoms Inventory Lipp (ISSL) was applied in Stage 1 and an interview was held to characterize the sample. In Stage 2, we applied again the ISSL and also the EPDS (Edinburgh Postnatal Depression Scale). Data were analyzed using SPSS for Windows ${ }^{\circledR}$, version 17.0. The statistical analyses were performed using the Student's ttest and the Spearman p. RESULTS: Seventy-eight percent of the participants showed significant signs of stress in the third quarter and $63 \%$ of them during the postpartum period, with a significant difference in the stress occurring in the third trimester and postpartum ( $t=2.20, p=0.03)$. There was also a correlation between the stress occurring during pregnancy and in the puerperium and the manifestation of postpartum depression $(p<0.001)$. CONCLUSION: More than half of the women experience significant stress signs during both pregnancy and the postpartum period. However, the frequency of onset of significant symptoms of stress was higher during pregnancy than during the puerperium. These results seem to be closely related to the manifestation of postpartum depression, indicating the relationship between stress and postpartum depression.
Programa de Pós-Graduação em Psicologia do Desenvolvimento e Aprendizagem. Faculdade de Ciências da Universidade Estadual Paulista "Julio de Mesquita Filho" - UNESP - Bauru (SP), Brasil.

"Professora Titular do Departamento de Psicologia da Universidade Estadual Paulista "Julio de Mesquita Filho" - UNESP - Bauru (SP), Brasil.

2 Aluna do Programa de Pós-Graduação em Psicologia do Desenvolvimento e Aprendizagem da Universidade Estadual Paulista "Julio de Mesquita Filho" - UNESP - Bauru (SP), Brasil. 
Introdução

O stress é um conjunto de respostas que o organismo emite para reagir frente a algo que o despertou. Quando o organismo é exposto frequentemente a situações que desencadeiam as reações de stress - os estressores - o corpo passa a trabalhar com gasto superior de energia. Pode ser entendido como uma resposta fisiológica, psicológica e comportamental de um indivíduo que procura se adaptar e se ajustar às solicitações internas e/ou externas ao organismo ${ }^{1}$. Em geral, a resposta ao stress é projetada para ter uma duração limitada e as mudanças resultantes desta atividade hormonal e de neurotransmissores são rapidamente restauradas aos níveis pré-stress. No entanto, quando o stress é de natureza crônica e o indivíduo não consegue se adaptar a ele, pode resultar em outros distúrbios, como ansiedade e depressão. Mudanças importantes na vida podem gerar respostas de stress nos sujeitos ${ }^{2}$.

O ciclo gravídico puerperal é marcado por alterações emocionais, frutos de fatores sociais e psicológicos, que podem influenciar o desenvolvimento da gestação, assim como o bem-estar e saúde materno-infantil. Entre os fatores psicológicos que, geralmente, implicam em complicação durante gestação, parto e pós-parto, estão os estressores vivenciados na gravidez e no puerpério ${ }^{3}$.

O stress na gestação, em geral, está associado a eventos específicos como enjoos, gravidez não planejada, medo de ganho excessivo de peso no início da gravidez e medo do parto, em meados da gestação. A média de eventos estressores durante a gestação é de cinco eventos por gestante ${ }^{4}$ e o quadro pode se agravar no contexto familiar se houver situação econômica difícil, violência doméstica, uso de drogas, depressão, pânico e complicações pré-natais ${ }^{5,6}$.

Pesquisas apontam que mais de $75 \%$ das gestantes apresentam sinais significativos de stress em algum nível ${ }^{5,6}$. Gestantes expostas por longo prazo a eventos estressores são fortes candidatas a apresentar riscos à sua saúde e a de seus descendentes ${ }^{7}$. Entre as consequências, o feto pode responder ao stress materno com predisposição a doenças mentais $^{8}$, alergias e asma ${ }^{9}$. Estudos têm também associado a presença de stress na gestação a prejuízos ao desenvolvimento infantil ${ }^{10,11}$. Quanto mais elevado o stress na gestação, a probabilidade de a criança apresentar problemas temperamentais e comportamentais ${ }^{12,13}$ aumenta, e problemas temperamentais na criança podem levar a mãe a sintomas depressivos ${ }^{14}$. O stress também pode resultar em sequelas danosas para a saúde do neonato, como a prematuridade abaixo do peso. O stress pode, também, causar complicações obstétricas na gestante ${ }^{15}$, além de ser um indicativo para manifestação de depressão pós-parto (DPP) ${ }^{16,17}$.

O puerpério, por si só, é um período estressante, de adaptação, durante o qual se desenrolam todas as manifestações involutivas ou de recuperação da genitália materna, que coincide com o período em que a mulher terá de reorganizar seu cotidiano, incluindo o bebê em sua dinâmica de vida. Consiste num período cronologicamente variável, de âmbito impreciso e, dependendo de como a mulher lida com ele, pode apresentar ou não sintomas significativos de stress.

A literatura a respeito do stress no puerpério é escassa, indicando a forte necessidade de que pesquisas sejam realizadas nessa área para melhor compreensão a respeito de seus indicadores, sua relação com o stress gestacional e possíveis influências do stress puerperal sobre o desenvolvimento infantil e sobre o comportamento materno ${ }^{18}$. O que se sabe, no momento, de forma ainda inconclusiva é que eventos estressores tanto na gestação como no puerpério podem ser indicativos para manifestação de sintomas de DPP ${ }^{16,17}$.

Os principais fatores de risco de DPP são: história anterior de depressão, dificuldades financeiras, baixa escolaridade, desemprego, ausência de suporte social, dependência de substâncias, violência doméstica e não aceitação da gravidez ${ }^{19-23}$. Pesquisas apontam que 10 a $20 \%$ das mulheres manifestam DPP 24,25 e no Brasil, esta porcentagem é ainda mais elevada ${ }^{22-27}$.

Até o momento, pesquisas nacionais e internacionais associando o stress apresentado tanto na gestação como no puerpério à manifestação de sintomas de DPP ainda são escassas. Entretanto, há pesquisas que indicam que eventos estressores vivenciados na gestação ou no puerpério são associados a manifestação de sintomas depressivos após o parto. Entre os eventos estressores estão: número de gestação, de partos, de filhos vivos, menor tempo de relacionamento com o parceiro, falta de rede de apoio, dificuldades financeiras, não aceitação da gravidez $z^{16,17,22-27}$.

O objetivo deste trabalho é o de preencher essa lacuna existente a respeito do stress no puerpério, a comparação entre os indicadores de stress na gestação e no puerpério e a correlação entre a manifestação de stress no ciclo gravídico puerperal e a manifestação de sintomas de DPP.

O objetivo do presente trabalho foi descrever e comparar o stress de primigestas no terceiro trimestre de gestação e no pós-parto, descrevendo as fases e o tipo dos sintomas, se físicos ou psicológicos. Pretendeu, também, averiguar a ocorrência de DPP, associando-os à manifestação de stress na gestação e no puerpério.

\section{Métodos}

Participaram da primeira etapa desta pesquisa 98 primigestas usuárias do Sistema Único de Saúde (SUS). A idade das participantes variou de 15 a 39 anos, sendo a idade média das participantes 21 anos. Quanto à 
escolaridade, $12 \%$ tinham ensino fundamental incompleto, $24 \%$ ensino fundamental completo, $54 \%$ ensino médio completo e $10 \%$ ensino superior completo. Em relação ao estado civil, $68 \%$ moravam com o parceiro (união estável ou civil), $15 \%$ estavam solteiras e $17 \%$ namorando. Sobre a ocorrência de aborto, $11 \%$ tiveram abortamento anterior à gestação atual e $10 \%$ relataram ameaça de aborto no início da gestação. Em relação à saúde durante a gestação, 86\% informaram ter apresentado boa saúde nesse período. Na segunda etapa da pesquisa, participaram 64 das 98 participantes da primeira etapa da pesquisa, o que caracteriza um estudo longitudinal.

Os dados da Etapa 1 foram coletados no Núcleo de Saúde Central de uma cidade do interior paulista e da Etapa 2, na residência das participantes, durante o período de junho de 2009 a janeiro de 2011.

Para avaliação de sintomas de stress utilizou-se o Inventário de Sintomas de Stress de Lipp (ISSL) ${ }^{28}$, para adultos com mais de 15 anos. O inventário é composto por três quadros, cada um deles se referindo à presença dos sintomas relacionados à distância temporal (quadro 1: 24 horas; quadro 2: 1 semana e quadro 3: 1 mês). Este instrumento pode ser aplicado em grupos de até 20 pessoas ou individualmente.

O ISSL contém 15 itens para o quadro 1 , sendo 12 itens relacionados a sintomas físicos e 3 relacionados a sintomas psicológicos; outros 15 itens para o quadro 2 , sendo 10 itens relacionados a sintomas físicos e 5 relacionados a sintomas psicológicos; e 23 itens para o quadro 3 , sendo 12 relacionados a sintomas físicos e 11 a sintomas psicológicos. As respostas foram assinaladas pelas participantes em cada quadro, com F1 que corresponde aos sintomas físicos ou P1 que corresponde aos sintomas psicológicos. A aplicação obedeceu às instruções do manual de aplicação.

Para avaliação de sintomas de DPP, utilizou-se a Escala de Edimburgo, adaptada da Edinburgh Postnatal Depression Scale ${ }^{29}$. A escala contém 10 afirmações que requerem que a participante descreva como se sente diante de situações do cotidiano, circulando o número apropriado à sua condição, de 0 a 3 , colocados à direita de cada afirmação na folha de respostas. A pontuação para Escala de Edimburgo adaptada varia de 1 mínimo de 0 a um máximo de 30 pontos.

Para a coleta dos dados, o setor de agendamento da UBS do centro possibilitou a identificação das primigestas cuja gestação estava no início do terceiro trimestre e os dias em que estas tinham consulta marcada com o obstetra ou ecografista. Nessa oportunidade eram convidadas a participar do projeto, recebendo as informações pertinentes, sobre as atividades que seriam realizadas em cada uma das etapas. Era esclarecido sobre o sigilo das informações por elas fornecidas quando da apresentação dos dados em eventos e publicações da área, a ausência de qualquer ônus para a participação na pesquisa, assim como da manutenção dos demais serviços por ela usufruídos no SUS, em caso de desistência do projeto, o que poderia ocorrer em qualquer fase. Redimidas todas as dúvidas e confirmado o aceite, assinaram um termo de consentimento livre e esclarecido.

Tal atividade ocorreu durante a espera para a consulta médica ou a realização da ultrassonografia. Ao fim da aplicação, estas foram informadas do resultado do ISSL e, ainda, encaminhadas, se necessário. Para todas explicou-se que haveria uma próxima etapa do presente projeto que ocorreria 45 dias após o nascimento do neonato e que a pesquisadora, de posse de telefones e endereços, entraria em contato com as que concordaram em participar dessa próxima etapa no puerpério.

Das 98 gestantes que participaram da primeira etapa da pesquisa, 64 (65\%) participaram também da segunda. As demais $(35 \%)$ não participaram desta segunda etapa por diversos motivos, tais como: falecimento do bebê após o nascimento; não aceitaram participar da segunda fase da pesquisa; detenção; mudança de cidade e/ou telefone.

No ISSL a tabulação dos dados foi realizada a partir das respostas das participantes que foram somadas por quadro: as respostas $\mathrm{P}$ (Psicológicos) e F (Físicos) separadamente, de modo que se tenham seis escores: três físicos e três psicológicos. Em seguida, somaramse os escores $\mathrm{P}+\mathrm{F}$ por quadro, de modo que se tiveram três escores, um para cada quadro do inventário. Se qualquer dos subescores de $\mathrm{P}+\mathrm{F}$ atingir o critério para o quadro pertinente, pode-se concluir que a pessoa tem stress e em que fase ela se encontra, se na de alerta, resistência, quase exaustão ou exaustão, conforme descrito no manual ${ }^{28}$.

A tabulação dos dados obtidos na EPDS foi feita somando-se os valores atribuídos pelas participantes para cada item. O critério utilizado baseou-se nos resultados da escala adaptada, cuja pontuação de 12 ou mais aponta para distúrbio depressivo de intensidade variável, indicando que a puérpera deve ser encaminhada ao profissional de saúde mental.

Os dados foram analisados usando o programa estatístico SPSS for Windows ${ }^{\circledR}$, versão 17.0, realizando-se análises estatística e descritiva. Para a comparação do stress nos dois momentos da pesquisa, durante a gestação e no puerpério, utilizou-se o Teste $t$ de Student para amostras pareadas (comparação dentre-grupos), adotando-se $\mathrm{p}<0,05$ como nível crítico. Para a correlação entre stress na gestação e no puerpério e manifestação de sintomas significativos de DPP utilizou-se a estatística p Spearman, adotando-se $\mathrm{p}<0,05$ como nível crítico.

O presente projeto foi submetido ao comitê de ética da Faculdade de Ciências da UNESP, campus de Bauru (Processo 
n614/46/01/09) e da Secretaria de Saúde de Bauru (Processo $\left.\mathrm{n}^{\circ} 16522 / 09\right)$, cuja aprovação significou a permissão para a coleta dos dados no referido núcleo de saúde.

\section{Resultados}

Os resultados indicaram que das 98 participantes, gestantes no terceiro trimestre de gestação, $76 \%(\mathrm{n}=78)$ manifestaram sintomas de stress. Delas, $72 \%(n=55)$ estavam na fase de resistência, 25\% $(\mathrm{n}=19)$ na fase de quase exaustão e 3\% ( $n=2)$ na fase de exaustão. Em relação aos sintomas de stress, $10 \%(\mathrm{n}=8)$ apresentaram sintomas de stress físico, $87 \%$ ( $\mathrm{n}=66)$, sintomas de stress psicológico e $3 \%(\mathrm{n}=2)$ apresentaram tanto stress físico como psicológico, concomitantemente.

Das 98 participantes, 64 participaram da segunda fase da pesquisa, no puerpério. Foram, então, computados os resultados obtidos no ISSL, no último trimestre da gestação e no puerpério dessas 64 participantes (Tabela 1).

Quando no terceiro trimestre da gestação, $75 \%(\mathrm{n}=48)$ manifestaram stress, sendo que delas, $73 \%(\mathrm{n}=35)$ estavam na fase de resistência, $25 \%(n=12)$ na fase de quase exaustão e $2 \%(n=1)$ na fase de exaustão. Em relação aos sintomas de stress, $13 \%(\mathrm{n}=6)$ apresentaram sintomas de stress físico, $85 \%(\mathrm{n}=41)$ sintomas de stress psicológico e $2 \%(\mathrm{n}=1)$ apresentaram tanto stress físico como psicológico, concomitantemente.

No puerpério, os resultados indicaram que 63\% $(\mathrm{n}=40)$ manifestaram sintomas de stress. Dessas participantes, $80 \%(n=32)$ estavam na fase de resistência e $20 \%$ $(\mathrm{n}=8)$ na fase de quase exaustão. Em relação aos sintomas de stress, $10 \%(\mathrm{n}=4)$ apresentaram sintomas de stress físico, $83 \%(n=33)$ sintomas de stress psicológico e $7 \%$ $(\mathrm{n}=3)$ apresentaram tanto stress físico como psicológico concomitantemente. A análise estatística conduzida (teste $t$ pareado) indicou que existe diferença significativa entre o stress manifestado pelas participantes na gestação e no puerpério $(t=2,2 ; \mathrm{p}<0,05)$, cujo número de participantes com stress diminuiu após o nascimento do bebê, bem como nas fases mais críticas.

A Tabela 2 apresenta a relação entre stress na gestação e no puerpério e sintomas de DPP. Das 43 gestantes avaliadas no terceiro trimestre, uma $(6 \%)$ das sem stress, $14(40 \%)$ das que estavam na fase de resistência, 5 (42\%) das que estavam na fase de quase exaustão e a única que estava na fase de exaustão foram classificadas com DPP. O teste $\mathrm{p}$ de Spearman indicou que existe correlação positiva $(\mathrm{p}=0,45 ; \mathrm{p}<0,001)$ entre os sintomas de stress apresentados na gestação e os sintomas de DPP e, quanto mais elevada a fase de stress no fim da gestação, mais elevada foi a pontuação na EPDS.

Em relação ao puerpério, $2(8 \%)$ das participantes sem stress, $13(41 \%)$ das que estavam na fase de resistência e
Tabela 1. Indicadores de stress na gestação e no puerpério

\begin{tabular}{lrrrr}
\hline & \multicolumn{2}{c}{ Gestação } & \multicolumn{2}{c}{ Puerpério } \\
\cline { 2 - 5 } & n & $\%$ & n & $\%$ \\
\hline Sem stress & 16 & 25 & 24 & 37 \\
Com stress & 48 & 75 & 40 & 63 \\
Resistência & 35 & 73 & 32 & 80 \\
Quase exaustão & 12 & 25 & 8 & 20 \\
Exaustão & 1 & 2 & 0 & 0 \\
\hline
\end{tabular}

Tabela 2. Relacionamento entre stress no terceiro trimestre de gestação e no puerpério e a manifestação de sintomas de depressão pós-parto

\begin{tabular}{|c|c|c|c|c|c|c|c|c|}
\hline & \multicolumn{4}{|c|}{ Gestação } & \multicolumn{4}{|c|}{ Puerpério } \\
\hline & \multicolumn{2}{|c|}{ Sem DPP } & \multicolumn{2}{|c|}{ Com DPP } & \multicolumn{2}{|c|}{ Sem DPP } & \multicolumn{2}{|c|}{ Com DPP } \\
\hline & n & $\%$ & n & $\%$ & n & $\%$ & n & $\%$ \\
\hline Sem stress & 15 & 94 & 1 & 6 & 22 & 92 & 2 & 8 \\
\hline Resistência & 21 & 60 & 14 & 40 & 19 & 59 & 13 & 41 \\
\hline Quase exaustão & 7 & 58 & 5 & 42 & 2 & 25 & 6 & 75 \\
\hline Exaustão & 0 & 0 & 1 & 100 & 0 & 0 & 0 & 0 \\
\hline Total & 43 & & 21 & & 43 & & 21 & \\
\hline
\end{tabular}

DPP: depressão pós-parto

$6(75 \%)$ das que estavam na fase de quase exaustão foram classificadas com DPP. O teste $\mathrm{p}$ de Spearman indicou que existe correlação positiva $(\mathrm{p}=0,6 ; \mathrm{p}<0,001)$ entre os sintomas de stress apresentados no puerpério e os sintomas de DPP e que quanto mais elevada a fase de stress no puerpério, mais elevada foi a pontuação na EPDS.

\section{Discussão}

No Brasil, há poucas pesquisas com gestantes que identifiquem o stress psicológico tanto no período pré-natal como no período pós-natal, sendo esta a primeira a comparar esses dois momentos e associá-los à presença da DPP. A depressão é uma doença que pode ser derivada do stress quando duradouro e em níveis elevados no organismo ${ }^{1}$.

Os resultados deste trabalho em relação ao stress indicaram que mais da metade das gestantes, no terceiro trimestre, apresentaram sintomas significativos, sendo que a fase mais frequente foi a de resistência, com prevalência de sintomas psicológicos. $\mathrm{O}$ índice de stress encontrado nesta pesquisa confirma os dados de outro trabalho ${ }^{5}$ brasileiro, que apresentou porcentagens também elevadas de mulheres com stress na gestação, sugerindo cuidados para que não evolua para níveis mais críticos.

O stress pode estar associado a eventos estressores próprios desta fase como os temores em relação ao parto, o medo de que ela ou o feto morra, preocupações financeiras, conjugais, falta de rede de apoio, dúvidas em relação ao corpo, entre vários outros motivos diferentes para cada gestante, principalmente se for a primeira gravidez. 
Em relação ao stress manifestado no puerpério, observou-se que mais da metade das participantes também foram classificadas com sintomas de stress, na fase de resistência, com prevalência de sintomas psicológicos. Pesquisas em relação ao stress no puerpério são escassas. Há grande número de trabalhos referentes ao stress na gestação, mas apenas um artigo foi encontrado referente ao stress no puerpério ${ }^{18}$. A saúde mental da mulher, nessa fase do ciclo vital, ainda é pouco explorada pelos pesquisadores que dão preferência aos estudos referentes à saúde mental da mulher no período gravídico. Dessa forma, é importante que pesquisadores deem atenção, também, aos aspectos da saúde mental da mulher no puerpério ${ }^{30}$. Um estudo recente indica taxas alarmantes de transtornos mentais em mulheres nessa fase pós-parto ${ }^{31}$.

Quando comparados à presença de stress antes e após o parto, os resultados revelaram que o stress é significativamente mais frequente no terceiro trimestre de gestação do que no puerpério. Mesmo que no puerpério a frequência de mulheres que manifestam stress seja inferior à da gestação, ainda assim, tem-se que mais da metade delas encontram-se estressadas. Desta forma, faz se importante que pesquisas sejam realizadas para identificar os possíveis fatores estressores potenciais no puerpério.

Os dados deste trabalho indicaram que a manifestação de stress tanto na gestação como no puerpério está relacionada à manifestação de DPP, sendo que quanto mais avançada a fase de stress em que a gestante ou a puérpera se encontrar, maior a probabilidade de esta apresentar DPP. Tal fato pode ser explicado, pois, quando a resposta do stress gera ativação fisiológica frequente e duradoura, pode provocar um esgotamento dos recursos do indivíduo, expondo-o a surgimento de transtornos psicofisiológicos diversos, predispondo ao aparecimento de depressão e outros problemas de saúde mental ${ }^{2}$.

Mais pesquisas em relação ao stress no ciclo gravídico puerperal devem ser realizadas utilizando o ISSL, pois é recente o uso desse instrumento para avaliar o stress nesta fase do ciclo vital. Os métodos de avaliação do stress nesta fase ainda são diversificados; alguns pesquisadores utilizam escalas, outros inventários, outros questionários e ainda outros medem o cortisol presente na saliva da mulher, correlacionando-os com variáveis diversas. Desta forma, a compreensão dos efeitos do stress na gestação e no puerpério sobre a saúde mental materna ainda é inconclusiva e limitada, já que as pesquisas apontaram diversas formas de medir o stress no ciclo gravídico-puerperal. Estudos associando o nível de cortisol e a aplicação do ISSL com gestantes e puérperas podem validar as duas medidas, correlacionando-as a variáveis sociodemográficas, por exemplo.

É importante ressaltar que há poucos estudos nacionais investigando o stress gestacional ${ }^{4,5}$, como na literatura estrangeira ${ }^{6}$, sendo que essa última tem apontado para os prejuízos que o stress pode causar no feto ${ }^{8-15}$ e, provavelmente, ao longo do ciclo vital do sujeito ${ }^{7,9-13}$ exposto na vida uterina ao stress materno. Quanto ao stress puerperal, trabalhos nacionais são escassos e há poucos trabalhos estrangeiros a respeito deste assunto ${ }^{16-18}$, indicando a forte necessidade de que mais pesquisas sejam realizadas a esse respeito.

A presente pesquisa apresentou resultados baseados em uma pequena população, limitando o estudo. Dessa forma, sugere-se que pesquisas sejam realizadas com uma amostra superior de mulheres, no fim da gestação e, depois, no puerpério, verificando a prevalência do stress nos dois momentos, relacionando as fases à manifestação de sintoma de DPP.

\section{Referências}

1. Margis R, Picon P, Cosner AF, Silveira RO. Relação entre estressores, estresse e ansiedade. Rev Psiquiatr Rio Gd Sul. 2003;25(Suppl1):65-74.

2. Santos AM, Castro JC. Stress. Anál Psicol. 1998;16(4):675-90.

3. Faisal-Cury A, Menezes, P, Arayar R, Zagaib M. Common mental disorders during pregnancy: prevalence and associated factors among low-income women in São Paulo, Brazil: depression and anxiety during pregnancy. Arch Womens Ment Health. 2009; 12(5):335-43.

4. Esper LH, Furtado EF. Associação de eventos estressores e morbidade psiquiátrica em gestantes. SMAD Rev Eletrônica Saúde Mental Álcool Drog. 2010;6 N Esp:368-86.

5. Segato L, Andrade A, Vasconcellos DIC, Matias TS, Rolim MKSB. Ocorrência e controle do estresse em gestantes sedentárias e fisicamente ativas. Rev Educ Fís/UEM. 2009;20(1):121-9.
6. Woods SM, Melville JL, Guo Y, Fan MY, Gavin A. Psychosocial stress during pregnancy. Am J Obstet Gynecol. 2010;202(1): 61.e1-7.

7. Rice F, Harold GT, Boivin J, van den Bree M, Hay DF, Thapar A. The links between prenatal stress and offspring development and psychopathology: disentangling environmental and inherited influences. Psychol Med. 2010;40(2):335-45.

8. DiPietro JA, Costigan KA, Gurewitsch ED. Fetal response to induced maternal stress. Early Hum Dev. 2003;74(2):125-38.

9. Cookson H, Granell R, Joinson C, Ben-Shlomo Y, Henderson AJ. Mother's anxiety during pregnancy associated with asthma in the children. J Allergy Clin Immunol. 2009;123(4):847-53.e 11

10. Davis EP, Sandman CA. The timing of prenatal exposure to maternal cortisol and psychosocial stress is associated with human infant cognitive development. Child Dev. 2010;81(1):131-48. 
11. Talge NM, Neal C, Glover V; Early Stress, Translational Research and Prevention Science Network: Fetal and Neonatal Experience on Child and Adolescent Mental Health. Antenatal maternal stress and long-term effects on child neurodevelopment: how and why? J Child Psychol Psychiatry. 2007;48(3-4):245-61.

12. Charil A, Laplante DP, Vaillancourt $C$, King S. Prenatal stress and brain development. Brain Res Rev. 2010;65(1):56-79.

13. Gutteling BM, de Weerth $C$, Willemsen-Swinkels SH, Huizink AC, Mulder EJ, Visser $G H$, et al. The effects of prenatal stress on temperament and problem behavior of 27-month-old toddlers. Eur Child Adolesc Psychiatry. 2005;14(1):41-51.

14. Britton JR. Infant temperament and maternal anxiety and depressed mood in the postpartum period. Women Health. $2011 ; 51$ (1):55-71.

15. Diego MA, Jones NA, Field T, Hernandez-Reif $M$, Schanberg $S$, Kuhn C, et al. Maternal psychological distress, prenatal cortisol, and fetal weight. Psychosom Med. 2006;68(5):747-53.

16. Brummelte $S$, Galea LA. Chronic corticosterone during pregnancy and postpartum affects maternal care, cell proliferation and depressivelike behavior in the dam. Horm Behav. 2010;58(5):769-79.

17. Gao LL, Chan SW, Mao Q. Depression, perceived stress, and social support among first-time Chinese mothers and fathers in the postpartum period. Res Nurs Health. 2009;32(1):50-8.

18. Meinlschmidt G, Martin C, Neumann ID, Heinrichs M. Maternal cortisol in late pregnancy and hypothalamic-pituitary-adrenal reactivity to psychosocial stress postpartum in women. Stress. 2010;13(2): 163-71.

19. Pereira PK, Lovisi GM. Prevalência da depressão gestacional e fatores associados. Rev Psiquiatr Clín. 2008;35(4):144-53.

20. Lancaster CA, Gold KJ, Flynn HA, Yoo H, Marcus SM, Davis MM. Risk factors for depressive symptoms during pregnancy: a systematic review. Am J Obstet Ginecol. 2010;202(1):5-14.

21. Mattar R, Silva EYK, Camano L, Abrahão AR, Colás OR, Andalaft Neto J, et al. A violência doméstica como indicador de risco no rastreamento de depressão pós-parto. Rev Bras Ginecol Obstet. 2007;29(9):470-7.

22. Moraes IGS, Pinheiro RT, Silva RA, Horta BL, Sousa PLR, Faria $A D$. Prevalência da depressão pós-parto e fatores associados. Rev Saúde Pública. 2006;40(1):65-70.

23. Gomes LA, Torquato VS, Feitoza AR, Souza AR, Silva MAM, Pontes RJS. Identificação dos fatores de risco para depressão pós-parto: importância do diagnóstico precoce. Rev RENE. 2010;11 N Esp: 1 17-23.

24. Hanna B, Jarman $H$, Savage $S$. The clinical application of three screening tools for recognizing post-partum depression. Int J Nurs Pract. 2004;10(2):72-9.

25. Righetti-Veltema M, Bousquet $A$, Manzano J. Impact of postpartum depressive symptoms on mother and her 18-month-old-infant. Eur Child Adolesc Psychiatry. 2003;12(2):75-83.

26. Fonseca VRJM, Silva GA, Otta E. Relação entre depressão pósparto e disponibilidade emocional materna. Cad Saúde Pública. 2010;26(4):738-46.

27. Ruschi GEC, Sun SY, Mattar R, Chambô Filho A, Zandonade E, Lima VJ. Aspectos epidemiológicos da depressão pós-parto em amostra brasileira. Rev Psiquiatr Rio Gd Sul. 2007;29(3):274-80.

28. Lipp MN. Manual do Inventário de Sintomas de Stress para Adultos de Lipp (ISSL). São Paulo: Casa do Psicólogo; 2000.

29. Santos IS, Matijasevich A, Tavares BF, Barros AJD, Botelho IP, Lapolli $C$, et al. Validation of the Edinburgh Postnatal Depression Scale (EPDS) in a simple of mothers from the 2004 Pelotas birth cohort study. Cad Saúde Pública. 2007;23(1 1):2577-88.

30. Cantilino A, Zambaldi CF, Sougey EB, Rennó Júnior J. Transtornos psiquiátricos no pós-parto. Rev Psiquiatr Clín. 2010;37(6):288-94.

31. Kerber SR, Falceto OG, Fernandes CLC. Problemas conjugais e outros fatores associados a transtornos psiquiátricos do pós-parto. Rev Bras Ginecol Obstet. 201 1;33(6):281-7. 